Article

\title{
Dissimilar Impact of a Mediterranean Diet and Physical Activity on Anthropometric Indices: A Cross-Sectional Study from the ILERVAS Project
}

\author{
Marta Sánchez ${ }^{1,+}$, Enric Sánchez ${ }^{1,+} \mathbb{D}$, Marta Hernández ${ }^{1} \mathbb{D}$, Jessica González ${ }^{2,3}$, \\ Francesc Purroy ${ }^{4}$, Ferran Rius ${ }^{1}$, Reinald Pamplona ${ }^{5}$, Cristina Farràs-Sallés ${ }^{6,7}$, \\ Liliana Gutiérrez-Carrasquilla ${ }^{1}$, Elvira Fernández ${ }^{8}$, Marcelino Bermúdez-López ${ }^{8}$, \\ Javier Salvador 9,10, Jordi Salas-Salvadó ${ }^{10,11} \mathbb{D}$, Albert Lecube $1,12, * \mathbb{D}$ and on behalf of the \\ ILERVAS project collaborators
}

1 Endocrinology and Nutrition Department, Arnau de Vilanova University Hospital, Obesity, Diabetes and Metabolism (ODIM) Research Group, IRBLleida, University of Lleida, 25198 Lleida, Spain; ma.san.pe.88@gmail.com (M.S.); esanchez@irblleida.cat (E.S.); martahernandezg@gmail.com (M.H.); frius.lleida.ics@gencat.cat (F.R.); liligutierrezc@gmail.com (L.G.-C.)

2 Respiratory Department, Arnau de Vilanova-Santa María University Hospital, Translational Research in Respiratory Medicine, IRBLleida, University of Lleida, 25198 Lleida, Spain; jegonzalez.lleida.ics@gencat.cat

3 Centro de Investigación Biomédica en Red de Enfermedades Respiratorias (CIBERES), Instituto de Salud Carlos III (ISCIII), 28029 Madrid, Spain

4 Stroke Unit, Arnau de Vilanova University Hospital, Clinical Neurosciences Group, IRBLleida, University of Lleida, 25198 Lleida, Spain; fpurroygarcia@gmail.com

5 Department of Experimental Medicina, IRBLleida, University of Lleida, 25198 Lleida, Spain; reinald.pamplona@mex.udl.cat

6 Applied Epidemiology Research Group, IRB Lleida, 25198 Lleida, Spain; cfarras.lleida.ics@gencat.cat

7 Unitat de Suport a la Recerca Lleida. Fundació Institut Universitari per a la recerca a l'Atenció Primària de Salut Jordi Gol i Gurina (IDIAPJGol), 08006 Barcelona, Spain

8 Vascular and Renal Translational Research Group, IRBLleida, RedinRen-ISCIII, 25198 Lleida, Spain; elvirafgiraldez@gmail.com (E.F.); mbermudez@irblleida.cat (M.B.-L.)

9 Endocrinology and Nutrition Department, Clínica Universidad de Navarra, 31008 Pamplona, Spain; jsalvador@unav.es

10 Human Nutrition Unit, Biochemistry and Biotechnology Department, University Hospital of Sant Joan de Reus, IISPV, Rovira i Virgili University, 43201 Reus, Spain; jordi.salas@urv.cat

11 Centro de Investigación Biomédica en Red de Fisiopatología de la Obesidad y Nutrición (CIBEROBN), Instituto de Salud Carlos III (ISCIII), 28029 Madrid, Spain

12 Centro de Investigación Biomédica en Red de Diabetes y Enfermedades Metabólicas Asociadas (CIBERDEM), Instituto de Salud Carlos III (ISCIII), 28029 Madrid, Spain

* Correspondence: alecube.lleida.ics@gencat.cat; Tel.: +34-973-70-51-83; Fax: +34-973-70-51-89

† Marta Sánchez and Enric Sánchez contributed equally to this work.

Received: 13 May 2019; Accepted: 14 June 2019; Published: 17 June 2019

check for updates

\begin{abstract}
There is a close relationship between lifestyle behaviors and excess adiposity. Although body mass index (BMI) is the most used approach to estimate excess weight, other anthropometric indices have been developed to measure total body and abdominal adiposity. However, little is known about the impact of physical activity and adherence to a Mediterranean diet on these indices. Here we report the results of a cross-sectional study with 6672 middle-aged subjects with low to moderate cardiovascular risk from the Ilerda Vascular (ILERVAS) project. The participants' adherence to physical activity (International Physical Activity Questionnaire short form) and MedDiet (Mediterranean Diet Adherence Screener) was evaluated. Measures of total adiposity (BMI, Clínica Universidad de Navarra-Body Adiposity Estimator (CUN-BAE), and Deurenberg's formula), central adiposity (waist and neck circumferences, conicity index, waist to height ratio, Bonora's equation, A body adiposity index, and body roundness index), and lean body mass (Hume formula) were
\end{abstract}


assessed. Irrespective of sex, lower indices of physical activity were associated with higher values of total body fat and central adiposity. This result was constant regardless of the indices used to estimate adiposity. However, the association between MedDiet and obesity indices was much less marked and more dependent on sex than that observed for physical activity. Lean body mass was influenced by neither physical activity nor MedDiet adherence. No joint effect between physical activity and MedDiet to lower estimated total or central adiposity indices was shown. In conclusion, physical activity is related to lower obesity indices in a large cohort of middle-aged subjects. MedDiet showed a slight impact on estimated anthropometric indices, with no joint effect when considering both lifestyle variables. ClinTrials.gov Identifier: NCT03228459.

Keywords: adiposity; body fat; Mediterranean diet; obesity indices; physical activity; questionnaire

\section{Introduction}

Obesity trends are increasing progressively and are especially influenced by changes in the lifestyle behaviors, especially a decrease of total physical activity and increase of caloric intake [1,2]. Obesity's growing prevalence is associated with a high social and economic impact, and according to the World Health Organization, there are more than 2.8 million annual deaths associated with diseases related to excess body weight [3]. In fact, adiposity excess has been linked with a wide range of diseases, including cardiovascular (CV) disease, cancer, type 2 diabetes, and sleep apnea-hypopnea syndrome [4]. Positively, even in moderate interventions, changes towards healthy habits-such as balanced diet and regular physical activity — have been reported to decrease body fat mass, improve lean body mass, and reduce overweight and obesity [5,6].

The traditional dietary pattern from populations bordering the Mediterranean Sea is characterized by an abundant consumption of extra-virgin olive oil, vegetables, fruits, legumes, and nuts and moderate consumption of fish and seafood, fermented dairy products, poultry, and red wine $[7,8]$. This pattern, the so-called Mediterranean diet (MedDiet), exerts potential benefits for certain types of cancer, type 2 diabetes, and some neurodegenerative diseases [9-11] and also prevents mortality from cardiovascular diseases $[12,13]$. MedDiet has been associated with lower total and regional adiposity in reproductive-aged women as well as with lower visceral and subcutaneous abdominal fat tissue in the Italian population [14,15]. Also, Barrea et al. have recently reported a novel association between the adherence to the MedDiet and phase angle, a direct measure by bioelectrical impedance analysis (BIA) used as a marker of cell membrane integrity and predictor of morbidity and survival in many diseases [16]. However, less is known about the role of MedDiet on total body fat and abdominal adiposity. On the other hand, physical activity practice also reduced body fat in middle-aged subjects, showing to be a stronger contributor to the variability in body fat than dietary habits $[5,6]$. Nevertheless, the associative and synergistic impact of the MedDiet and physical activity on anthropometric indices related to body composition has not been previously evaluated.

In clinical and epidemiological studies, body mass index (BMI) is widely used to assess the presence of obesity. However, BMI classification is far from perfect for providing an accurate measurement of body fat amount and distribution [17,18]. However, the gold standard techniques to directly assess total adipose tissue and abdominal adiposity, such as dual-energy $\mathrm{x}$-ray absorptiometry (DXA) and magnetic resonance imaging, still show a limited widespread in clinical practice, largely because of their complexity, high cost, and time-consuming protocols [19]. For this reason, several mathematical indices that combine traditional anthropometric measures have been developed to estimate total body fat and abdominal adiposity [20-26]. However, little is known regarding the impact of MedDiet and physical activity on these estimated measurements.

On this basis, the main objective of this study was to appraise the associations between adherence to the MedDiet and intensity of physical activity practice, as well as their simultaneous influence on 
adiposity estimated by different indices in a large cohort of middle-aged overweight participants. For this purpose, we applied two validated questionnaires, three indices of total adiposity, seven central adiposity measurements, and one lean body mass formula. The study was done according to sex, as its disparity of adiposity distribution and body fatness has been well documented [27].

\section{Materials and Methods}

\subsection{Design of the Study and Description of the Study Population}

A total of 6672 subjects were enrolled between 2015 and 2017 from 29 primary health care centers in the ongoing Ilerda Vascular (ILERVAS) study dealing with the subclinical atheromatous disease in Lleida, Catalonia, Spain (ClinTrials.gov Identifier: NCT03228459) [28]. Participants with an open history in the electronic primary care medical history database (e-CAP) were selected through simple randomization without replacement, within clusters defined by basic healthcare area. The participant selection process is shown in Supplemental Figure S1. The inclusion criteria were as follows: men and women between 45 and 70 years with at least one cardiovascular risk factor (hypertension, smoking, obesity, dyslipidemia, or having a first-degree relative with premature cardiovascular disease). The exclusion criteria were the presence of any type of diabetes, chronic kidney disease, cardiovascular disease (angina, myocardial infarction, stroke, peripheral artery disease, heart failure, history of vascular surgery), active neoplasia, institutionalized population, a life expectancy of less than 18 months, and pregnancy. A severe functional limitation or a significant cognitive deterioration were not considered exclusion criteria except in the case that they prevented the participants from reaching the place where the anthropometric measures were performed or responding adequately to the administered questionnaires. The prescribed antihypertensive, lipid-lowering, and antithrombotic treatments were acquired from prescription- and pharmacy-invoicing databases provided by the Catalan Health Service, which are incorporated yearly into the information system for the development of research in primary care database.

\subsection{Adherence to the Mediterranean Diet Assessment}

To quantitatively assess the adherence to the MedDiet, the validated 14-item Mediterranean Diet Adherence Screener (MEDAS) was used. This questionnaire was developed to rapidly control the compliance with the dietary intervention in the Prevención con Dieta Mediterránea (PREDIMED) trial $[19,29]$. The frequencies of consumption of olive oil, wine, fruits, vegetables, fish, legumes, sofrito (a Mediterranean sauce made with virgin olive oil, tomato, onions, garlic, and species), and nuts were evaluated. The intake of meat or meat-products, butter, bakery products, and sweetened soft beverages per week was also involved in the composite score. Information was obtained through self-reported data, and the final score ranged from 0 to 14 and classified participants according to their level of adherence to MedDiet: high (score $\geq 11$ points), moderate (7-10 points), and low ( $\leq 6$ points) adherence [11].

\subsection{Physical Activity Intensity and Type Assessment}

The short version of The International Physical Activity Questionnaire (IPAQ) was administered to all participants. This questionnaire was developed to evaluate physical activity and inactivity in adults [30]. The IPAQ short form asks questions about three detailed types of activity undertaken in four domains: leisure time, domestic and gardening, work-related, and transport-related physical activity. The detailed types of activity that were assessed were walking, moderate-intensity, and vigorous-intensity actions. The metabolic equivalent of task (METs)-min per week, a multiple of the resting metabolic rate, was assessed. Participants were classified following the IPAQ procedure into vigorous, moderate, and low physical activity [30]. The vigorous group was defined as those subjects with vigorous intensity activity on $\geq 3$ days achieving a minimum total physical activity of at least 1500 MET-min per week, or $\geq 7$ days of any combination of physical activity achieving $\geq 3000$ MET-min per 
week. Those who scored moderately on the IPAQ engaged in $\geq 3$ days of vigorous intensity activity and/or walking of at least $30 \mathrm{~min}$ per day, or $\geq 5$ days of moderate intensity activity and/or walking of at least $30 \mathrm{~min}$ per day, or $\geq 5$ days of any combination achieving $\geq 600$ METs min per week. Those participants with any of the criteria for either moderate or high levels of physical activity were classified as the low level of physical activity group [30].

\subsection{Assessment of Obesity-Related Parameters}

Weight and height were measured in light clothing and without shoes using standard equipment, to the nearest $0.5 \mathrm{~kg}$ and $1.0 \mathrm{~cm}$, respectively. BMI was calculated as the ratio of body weight $(\mathrm{kg})$ to the height (m) squared [31]. A non-stretchable tape with an accuracy of $0.1 \mathrm{~cm}$ was used to evaluate waist and neck circumferences. Waist circumference was measured midway between the lowest rib and the iliac crest on the horizontal plane with the subject in a standing position [32]. Neck circumference was measured in a plane as horizontal as possible, immediately below the laryngeal prominence, while standing erect with eyes facing forward [33]. All measurements were performed under strictly standardized conditions by one experienced nurse, using the same device to avoid inter-observer and inter-device variability. The relative intra-evaluator technical error measurement was lower than $1 \%$ for height, weight, waist, and neck circumferences. As the hip circumference was not measured in our population, anthropometric indices, including hip circumference, were not considered in our study.

Two equations were added to BMI to estimate total body fat: the Clínica Universidad de Navarra-Body Adiposity Estimator (CUN-BAE) and the formula proposed by Deurenberg et al. [20,21]. The CUN-BAE was calculated as follow: $-44.988+(0.503 \times$ age $)+(10.689 \times$ sex $)+(3.172 \times$ BMI $)-$ $\left(0.026 \times \mathrm{BMI}^{2}\right)+(0.181 \times \mathrm{BMI} \times \operatorname{sex})-(0.02 \times \mathrm{BMI} \times$ age $)-\left(0.005 \times \mathrm{BMI}^{2} \times \operatorname{sex}\right)+\left(0.00021 \times \mathrm{BMI}^{2} \times\right.$ age), where male is 0 and female is 1 for sex, and age in years. The Deurenberg equation assessed body fat using the formula: $(1.20 \times \mathrm{BMI})+(0.23 \times$ age $)-(10.8 \times$ sex $)-5.4$, where sex is 0 for females and 1 for males.

Five equations were added to the waist and neck circumferences to estimate central adiposity: (i) the conicity index proposed by Valdez et al. in 1991, (ii) the waist to height ratio, (iii) the Bonora's equation, (iv) the A body adiposity index developed by Krakauer et al. in 2012, and (v) the body roundness index proposed more recently by Thomas et al. [22-26]. The conicity index is based on the hypothesis that people that accumulate abdominal fat have a silhouette like a double cone (that is, two cones sharing the same base, one positioned over the other), whereas subjects with less visceral fat have the shape of a cylinder [22]. Therefore, conicity index ranges from 1.73 (a perfect double cone) to 1.0 (a perfect cylinder) and was obtained following the next equation: $0.109^{-1} \times$ waist circumference $(\mathrm{m}) \times(\text { weight }(\mathrm{kg}) / \text { height }(\mathrm{m}))^{-1 / 2}$ [22]

The waist to height ratio was calculated as waist circumference $(\mathrm{m})$ divided by height $(\mathrm{m})$ [23]. The equation proposed by Bonora et al. uses different formulas according to gender: $-453.7+(6.37 \times$ waist circumference $)$ for men, and $-370.5+(4.04 \times$ waist circumference $)+(2.62 \times$ age $)$ for women [24] . To obtain the A body shape index, the eccentricity of the body $(\varepsilon)$ is determined first [25]. The $\varepsilon$ is a non-dimensional value that quantifies the degree of circularity of an ellipse, ranges from zero (perfect circle) to one (a vertical line) and is calculated by the formula: $\left(1-\pi^{-2} \times\right.$ waist circumference $(\mathrm{m})^{2} \times$ height $\left.(\mathrm{m})^{-2}\right)^{1 / 2}$. Subsequently, the next formula calculates the index: $364.2-(365.5 \times \varepsilon)$, in which values closer to 1 are related to rounder individuals, whereas lower values are associated with leaner individuals [25]. Finally, the body roundness index was calculated as: waist circumference $(\mathrm{m}) /\left(\mathrm{BMI}^{2 / 3}\right.$ $\times$ height $\left.(\mathrm{m})^{1 / 2}\right)[26]$.

Finally, the Hume formula was applied to estimate lean body mass: $0.32810 \times$ weight $(\mathrm{kg})+$ $0.33929 \times$ height $(\mathrm{cm})-29.5336$ (for males), and $0.29569 \times$ weight $(\mathrm{kg})+0.41813 \times$ height $(\mathrm{cm})-$ 43.2933 (for females) [34]. 


\subsection{Covariates Assessment}

The smoking status (never/current/former) was recorded. Smokers who stopped smoking $\geq 1$ year prior to recruitment were considered former smokers. Blood pressure was measured in triplicate, after 5 min of rest using an automated device (Omron M6 Comfort HEM-7221-E (Omron Healthcare, Kyoto, Japan)) at 2-min intervals, and the mean of the last two was calculated. A dried capillary blood sample was used for the total cholesterol $\left(\mathrm{mg} / \mathrm{dL}\right.$ ) measurement using a REFLOTRON ${ }^{\circledR}$ (Roche Diagnostics, GmbH, Germany).

\subsection{Ethical Approval}

Informed consent was obtained from all subjects involved in the study. The procedure was approved by the Arnau de Vilanova University Hospital ethics committee (CEIC-1410). Moreover, the study was also completed according to the ethical guidelines of the Helsinki Declaration and Spanish regulation concerning the protection of personal data.

\subsection{Statistical Analysis}

Subjects were classified based on the adherence to both MedDiet (low/moderate/high) and physical activity practice (low/moderate/vigorous). The normal distribution of the variables was evaluated using the Shapiro-Wilk test. Given its skewed distribution, quantitative data were expressed as the median [interquartile range]. Baseline characteristics across categories were compared by the Kruskal-Wallis test (with a Bonferroni post-hoc analysis) and the Pearson's Chi-squared test for continuous and categorical variables, respectively. Also, two-way ANOVA was used to determine how obesity indices were affected by the adherence to both physical activity and MedDiet together. $p$-values $<0.05$ were considered statistically significant. All statistical tests were done using the SSPS statistical package (IBM SPSS Statistics for Windows, Version 25.0. Armonk, NY, USA).

\section{Results}

Most participants in the ILERVAS project showed a low physical activity practice (66.0\%) and a moderate adherence to MedDiet (75.7\%) (Tables 1 and 2). Subjects with low physical activity practice were mainly men, older, with a higher BMI, as well as a higher prevalence of hypertension and hypercholesterolemia than participants who referred to practice a vigorous physical activity (Table 1). In both genders, lower indices of physical activity were associated with higher values of total body fat and central adiposity (Table 3). This result was constant regardless of the indices used to estimate adiposity. However, physical activity practice was not related to lean body mass estimated by the Hume formula.

Participants with lower adherence to MedDiet were mainly men, younger, with a higher BMI, and a higher prevalence of smoking history than subjects who referred to higher adherence to diet (Table 2). Curiously, the lower adherence to MedDiet was also associated with a lower prevalence of hypertension and dyslipidemia. The association between adherence to MedDiet and obesity indices was much less marked and more dependent on sex than that observed when analyzing physical activity (Table 4). Male participants with higher adherence to MedDiet showed lower values of central adiposity (conicity index, waist to height ratio, A body shape index, and body roundness index) than participants with moderate adherence ( $p \leq 0.036$ for all), without differences in total adiposity indices. Contrary, female participants with high adherence to MedDiet presented lower total (BMI and CUN-BAE) and central adiposity (waist to hip ratio and body roundness index) measurements in comparison to those females with moderate adherence to MedDiet. 
Table 1. Main clinical characteristics and comorbidities of the entire study population according to their physical activity practice.

\begin{tabular}{cccccc}
\hline & Low PA & Moderate PA & Vigorous PA & $p^{*}$ & $p^{* *}$ \\
\hline Number $(\%)$ & $4408(66.0)$ & $1884(28.2)$ & $380(5.6)$ & - & - \\
Women, $n(\%)$ & $2137(48.5)$ & $1192(63.3)$ & $97(25.5)$ & $<0.001$ & $<0.001$ \\
Age (years) & $57(52 ; 62)$ & $59(54 ; 64)$ & $54(50 ; 60)$ & $<0.001$ & $<0.001$ \\
Current or former smoker, $n(\%)$ & $2704(61.3)$ & $992(52.7)$ & $220(57.0)$ & 0.186 & 0.062 \\
Hypertension, $n(\%)$ & $1764(40.0)$ & $778(41.3)$ & $125(32.9)$ & 0.006 & 0.002 \\
Systolic blood pressure (mmHg) & $131(120 ; 142)$ & $129(119 ; 142)$ & $127(117 ; 137)$ & 0.091 & 0.768 \\
Diastolic blood pressure (mmHg) & $82(75 ; 88)$ & $81(75 ; 87)$ & $81(75 ; 87)$ & 0.146 & 1.000 \\
Antihypertensive drugs, $n(\%)$ & $1464(33.2)$ & $660(35.0)$ & $101(26.6)$ & 0.008 & 0.001 \\
Dyslipidemia, $n(\%)$ & $2276(51.6)$ & $1031(54.7)$ & $203(53.4)$ & 0.503 & 0.642 \\
Total cholesterol $\geq 200 \mathrm{mg} / \mathrm{dL}, n(\%)$ & $2385(54.1)$ & $1086(57.6)$ & $196(51.6)$ & 0.343 & 0.030 \\
Lipid-lowering agents, $n(\%)$ & $797(18.1)$ & $381(20.2)$ & $71(18.7)$ & 0.770 & 0.494 \\
Obesity, $n(\%)$ & $1361(30.9)$ & $561(29.8)$ & $78(20.5)$ & $<0.001$ & $<0.001$ \\
BMI $\left(\mathrm{kg} / \mathrm{m}^{2}\right)$ & $28.8(25.9 ; 32.2)$ & $28.3(25.4 ; 31.6)$ & $27.7(25.1 ; 30.2)$ & $<0.001$ & 0.002 \\
Antithrombotic drugs, $n(\%)$ & $138(3.1)$ & $57(3.0)$ & $13(3.4)$ & 0.756 & 0.684 \\
\hline
\end{tabular}

${ }^{*}$ High vs. Low PA ** High vs. Moderate PA. Data are expressed as median (interquartile range) or $n$ (percentage). PA: Physical activity; BMI: body mass index. Antihypertensive drugs include angiotensin-converting enzyme (ACE) inhibitors, diuretics, angiotensin-II receptor antagonists (ARA II), beta-blockers, calcium antagonists, and other antihypertensives. Lipid-lowering treatments involve statins, fibrates, ezetimibe, and omega- 3 fatty acids. Antithrombotic drugs include anticoagulants and antiplatelets.

Table 2. Main clinical characteristics and comorbidities of the study population according to their adherence to the Mediterranean diet.

\begin{tabular}{cccccc}
\hline & Low MedDiet & $\begin{array}{c}\text { Moderate } \\
\text { MedDiet }\end{array}$ & High MedDiet & $p^{*}$ & $p^{* *}$ \\
\hline Number (\%) & $1076(16.1)$ & $5054(75.7)$ & $542(8.1)$ & - & - \\
Women, $n(\%)$ & $419(38.9)$ & $2695(53.3)$ & $213(57.6)$ & $<0.001$ & 0.060 \\
Age (years) & $55(51 ; 60)$ & $58(53 ; 63)$ & $58(54 ; 64)$ & $<0.001$ & 0.240 \\
Current or former smoker, $n(\%)$ & $763(70.9)$ & $2896(57.3)$ & $257(47.4)$ & $<0.001$ & $<0.001$ \\
Hypertension, $n(\%)$ & $397(36.9)$ & $2036(40.3)$ & $234(43.2)$ & 0.015 & 0.193 \\
Systolic blood pressure (mmHg) & $129(119 ; 142)$ & $130(120 ; 142)$ & $129(119 ; 137)$ & 0.257 & 0.257 \\
Diastolic blood pressure (mmHg) & $82(76 ; 88)$ & $81(75 ; 88)$ & $80(74 ; 87)$ & 0.003 & 0.093 \\
Antihypertensives, $n(\%)$ & $333(30.9)$ & $1710(33.8)$ & $182(33.6)$ & 0.284 & 0.905 \\
Dyslipidemia, $n(\%)$ & $512(47.6)$ & $2686(53.1)$ & $312(57.6)$ & $<0.001$ & 0.050 \\
Total cholesterol $\geq 200 \mathrm{mg} / \mathrm{dL}, n(\%)$ & $572(53.2)$ & $2781(55.0)$ & $314(57.9)$ & 0.069 & 0.196 \\
Lipid-lowering agents, $n(\%)$ & $187(17.4)$ & $958(19.0)$ & $104(19.2)$ & 0.371 & 0.895 \\
Obesity, $n(\%)$ & $213(29.0)$ & $3515(30.5)$ & $149(27.5)$ & 0.527 & 0.154 \\
BMI $\left(\mathrm{kg} / \mathrm{m}^{2}\right)$ & $28.7(25.5 ; 32.0)$ & $28.7(25.8 ; 32.0)$ & $27.9(25.2 ; 31.4)$ & 0.041 & 0.001 \\
Antithrombotic drugs, $n(\%)$ & $35(3.3)$ & $158(3.1)$ & $15(2.8)$ & 0.594 & 0.647 \\
\hline
\end{tabular}

* High vs. Low adherence to Mediterranean diet ${ }^{* *}$ High vs. Moderate adherence to Mediterranean diet. Data are expressed as median [interquartile range] or n (percentage). MedDiet: Mediterranean diet; BMI: body mass index. Antihypertensive drugs include angiotensin-converting enzyme (ACE) inhibitors, diuretics, angiotensin-II receptor antagonists (ARA II), beta-blockers, calcium antagonists, and other antihypertensives. Lipid-lowering treatments involve statins, fibrates, ezetimibe, and omega-3 fatty acids. Antithrombotic drugs include anticoagulants and antiplatelets.

Finally, Table 5 exhibits the interactions between physical activity and MedDiet adherence with adiposity indices: a positive association was detected in both genders, but not observed when adherence of MedDiet was included in the joint analysis. 
Table 3. Obesity indices in male and female participants according to their physical activity practice.

\begin{tabular}{|c|c|c|c|c|c|}
\hline MALE SUBJECTS & Low PA & Moderate PA & Vigorous PA & $p^{*}$ & $p^{* *}$ \\
\hline$n$ & 2270 & 692 & 283 & - & - \\
\hline \multicolumn{6}{|l|}{ Total adiposity } \\
\hline BMI $\left(\mathrm{Kg} / \mathrm{m}^{2}\right)$ & $29.0(26.4 ; 32.0)$ & $28.6(26.0 ; 31.3)$ & $27.8(25.4 ; 30.2)$ & $<0.001$ & 0.004 \\
\hline CUN-BAE (\%) & $30.7(27.3 ; 34.2)$ & $30.2(27.0 ; 33.8)$ & $29.0(25.8 ; 32.3)$ & $<0.001$ & $<0.001$ \\
\hline Deurenberg $(\%)$ & $31.4(28.0 ; 35.0)$ & $31.0(27.8 ; 35.1)$ & $29.3(26.4 ; 32.8)$ & $<0.001$ & $<0.001$ \\
\hline \multicolumn{6}{|l|}{ Central adiposity } \\
\hline Waist circumference $(\mathrm{cm})$ & $102(96 ; 110)$ & $101(94 ; 108)$ & $98(90 ; 104)$ & $<0.001$ & $<0.001$ \\
\hline Conicity index & $1.34(1.30 ; 1.38)$ & $1.33(1.29 ; 1.38)$ & $1.30(1.25 ; 1.30)$ & $<0.001$ & $<0.001$ \\
\hline Waist to height ratio & $0.60(0.56 ; 0.64)$ & $0.59(0.55 ; 0.64)$ & $0.57(0.53 ; 0.61)$ & $<0.001$ & $<0.001$ \\
\hline Bonora $\left(\mathrm{cm}^{2}\right)$ & $196(158 ; 247)$ & $190(145 ; 234)$ & $171(120 ; 209)$ & $<0.001$ & $<0.001$ \\
\hline A body shape index & $\begin{array}{c}0.083 \\
(0.081 ; 0.085)\end{array}$ & $\begin{array}{c}0.083 \\
(0.081 ; 0.085)\end{array}$ & $\begin{array}{c}0.081 \\
(0.079 ; 0.084)\end{array}$ & $<0.001$ & $<0.001$ \\
\hline Body roundness index & $5.34(4.54 ; 6.34)$ & $5.25(4.36 ; 6.24)$ & $4.63(3.92 ; 5.61)$ & $<0.001$ & $<0.001$ \\
\hline Neck circumference $(\mathrm{cm})$ & $41.0(39.0 ; 43.0)$ & $40.5(39.0 ; 42.5)$ & $40.0(38.0 ; 42.0)$ & $<0.001$ & 0.048 \\
\hline \multicolumn{6}{|l|}{ Lean body mass } \\
\hline Hume (kg) & $56.4(52.5 ; 60.4)$ & $55.4(51.6 ; 59.2)$ & $55.6(52.2 ; 59.6)$ & 0.197 & 1 \\
\hline \multicolumn{6}{|l|}{ FEMALE SUBJECTS } \\
\hline$n$ & 2137 & 1192 & 97 & - & - \\
\hline \multicolumn{6}{|l|}{ Total adiposity } \\
\hline BMI $\left(\mathrm{Kg} / \mathrm{m}^{2}\right)$ & $28.7(25.3 ; 32.5)$ & $28.1(25.2 ; 31.9)$ & $27.0(24.6 ; 30.1)$ & 0.006 & 0.081 \\
\hline CUN-BAE (\%) & $42.5(38.4 ; 46.4)$ & $42.0(38.4 ; 45.9)$ & $40.4(37.2 ; 43.6)$ & 0.004 & 0.036 \\
\hline Deurenberg $(\%)$ & $32.1(27.7 ; 36.8)$ & $31.6(27.6 ; 36.1)$ & $29.4(26.6 ; 33.4)$ & 0.003 & 0.018 \\
\hline \multicolumn{6}{|l|}{ Central adiposity } \\
\hline Waist circumference $(\mathrm{cm})$ & $100(92 ; 108)$ & $98(90 ; 106)$ & $96(89 ; 103)$ & 0.008 & 0.312 \\
\hline Conicity index & $1.36(1.31 ; 1.41)$ & $1.35(1.30 ; 1.40)$ & $1.33(1.30 ; 1.39)$ & 0.04 & 0.641 \\
\hline Waist to height ratio & $0.64(0.58 ; 0.69)$ & $0.62(0.58 ; 0.68)$ & $0.60(0.57 ; 0.65)$ & $<0.001$ & 0.046 \\
\hline Bonora $\left(\mathrm{cm}^{2}\right)$ & $188(153 ; 224)$ & $183(150 ; 218)$ & $170(140 ; 202)$ & 0.004 & 0.051 \\
\hline A body shape index & $0.085(0.082 ; 0.088)$ & $0.084(0.081 ; 0.087)$ & $0.084(0.081 ; 0.087)$ & 0.69 & 1 \\
\hline Body roundness index & $6.17(5.02 ; 7.46)$ & $5.92(4.87 ; 7.20)$ & $5.45(4.62 ; 6.60)$ & $<0.001$ & 0.046 \\
\hline Neck circumference $(\mathrm{cm})$ & $35.0(33.5 ; 37.0)$ & $35.0(33.0 ; 36.5)$ & $34.5(33.0 ; 36.0)$ & 0.043 & 0.586 \\
\hline \multicolumn{6}{|l|}{ Lean body mass } \\
\hline Hume $(\mathrm{kg})$ & $43.3(39.8 ; 46.8)$ & $42.5(39.3 ; 46.2)$ & $42.4(39.9 ; 46.1)$ & 1 & 1 \\
\hline
\end{tabular}

${ }^{*}$ Low vs. High PA ${ }^{* *}$ Moderate vs. High PA. Data are expressed as a median [interquartile range]. PA: physical activity; BMI: body mass index; CUN-BAE: Clinica Universidad de Navarra-Body Adiposity Estimator.

Table 4. Results of the obesity indices in male and female participants according to their adherence to the Mediterranean diet.

\begin{tabular}{cccccc}
\hline MALE SUBJECTS & Low MedDiet & $\begin{array}{c}\text { Moderate } \\
\text { MedDiet }\end{array}$ & High MedDiet & $p^{*}$ & $p^{* *}$ \\
\hline$n$ & 657 & 2359 & 230 & - & - \\
\hline Total adiposity & & & & \\
\hline BMI (Kg/m $\left.{ }^{2}\right)$ & $28.7(25.9 ; 31.6)$ & $28.9(26.4 ; 31.7)$ & $28.1(25.8 ; 31.5)$ & 1 & 0.101 \\
CUN-BAE (\%) & $30.1(26.6 ; 33.7)$ & $30.6(27.3 ; 34.0)$ & $29.5(26.6 ; 33.8)$ & 1 & 0.138 \\
Deurenberg (\%) & $30.5(27.2 ; 34.5)$ & $31.3(28.0 ; 34.9)$ & $30.2(27.3 ; 34.9)$ & 1 & 0.246 \\
\hline Central adiposity & & & & \\
\hline Waist circumference $(\mathrm{cm})$ & $101(94 ; 109)$ & $102(96 ; 109)$ & $100(94 ; 107)$ & 0.057 & 0.057 \\
Conicity index & $1.33(1.29 ; 1.38)$ & $1.33(1.30 ; 1.38)$ & $1.32(1.28 ; 1.36)$ & 0.164 & 0.015 \\
Waist to height ratio & $0.59(0.55 ; 0.64)$ & $0.60(0.56 ; 0.64)$ & $0.58(0.55 ; 0.63)$ & 0.592 & 0.023 \\
Bonora (cm $\left.{ }^{2}\right)$ & $190(145 ; 241)$ & $196(158 ; 241)$ & $183(145 ; 228)$ & 0.06 & 0.06 \\
A body shape index & 0.083 & 0.083 & 0.082 & 0.101 & 0.036 \\
Body roundness index & $(0.081 ; 0.085)$ & $(0.081 ; 0.085)$ & $(0.080 ; 0.085)$ & 0.592 & 0.023 \\
Neck circumference $(\mathrm{cm})$ & $5.22(4.26 ; 6.23)$ & $5.29(4.49 ; 6.26)$ & $4.97(4.31 ; 6.13)$ & 0.176 & 0.176 \\
\hline Lean body mass & $40.5(39.0 ; 42.5)$ & $41.0(39.0 ; 43.0)$ & $40.5(39.0 ; 43.0)$ & & \\
\hline Hume $(\mathrm{kg})$ & $56.5(52.3 ; 60.1)$ & $56.0(52.1 ; 60.2)$ & $55.9(52.6 ; 59.9)$ & 0.594 & 0.594 \\
\hline
\end{tabular}


Table 4. Cont.

\begin{tabular}{|c|c|c|c|c|c|}
\hline FEMALE SUBJECTS & & & & & \\
\hline$n$ & 419 & 2695 & 312 & - & - \\
\hline \multicolumn{6}{|l|}{ Total adiposity } \\
\hline BMI $\left(\mathrm{Kg} / \mathrm{m}^{2}\right)$ & $28.5(24.9 ; 32.8)$ & $28.5(25.3 ; 32.3)$ & $27.8(24.9 ; 31.1)$ & 0.077 & 0.02 \\
\hline CUN-BAE (\%) & $42.3(37.9 ; 46.6)$ & $42.4(38.5 ; 46.3)$ & $41.7(37.8 ; 45.3)$ & 0.285 & 0.041 \\
\hline Deurenberg $(\%)$ & $31.7(26.9 ; 36.8)$ & $32.0(27.8 ; 36.6)$ & $31.3(27.1 ; 35.6)$ & 0.07 & 0.07 \\
\hline \multicolumn{6}{|l|}{ Central adiposity } \\
\hline Waist circumference $(\mathrm{cm})$ & $99(90 ; 107)$ & $99(91 ; 107)$ & $98(90 ; 105)$ & 0.087 & 0.087 \\
\hline Conicity index & $1.35(1.31 ; 1.40)$ & $1.36(1.30 ; 1.41)$ & $1.36(1.30 ; 1.40)$ & 0.465 & 0.465 \\
\hline Waist to height ratio & $0.63(0.58 ; 0.68)$ & $0.63(0.58 ; 0.69)$ & $0.62(0.57 ; 0.68)$ & 0.441 & 0.024 \\
\hline Bonora $\left(\mathrm{cm}^{2}\right)$ & $182(144 ; 218)$ & $187(153 ; 222)$ & $182(151 ; 215)$ & 0.082 & 0.082 \\
\hline A body shape index & $0.084(0.081 ; 0.087)$ & $0.085(0.081 ; 0.088)$ & $0.085(0.082 ; 0.088)$ & 0.156 & 0.156 \\
\hline Body roundness index & $6.14(4.84 ; 7.24)$ & $6.07(5.00 ; 7.38)$ & $5.73(4.79 ; 7.14)$ & 0.441 & 0.024 \\
\hline Neck circumference $(\mathrm{cm})$ & $35.0(33.5 ; 37.0)$ & $35.0(33.5 ; 37.0)$ & $34.5(33.0 ; 36.5)$ & 0.015 & 0.149 \\
\hline \multicolumn{6}{|l|}{ Lean body mass } \\
\hline Hume $(\mathrm{kg})$ & $43.7(39.8 ; 47.0)$ & $42.9(39.6 ; 46.5)$ & $41.9(39.5 ; 46.3)$ & 0.095 & 0.095 \\
\hline
\end{tabular}

* Low vs. High PA ** Moderate vs. High PA. Data are expressed as a median (interquartile range). MedDiet: Mediterranean diet; BMI: body mass index; CUN-BAE: Clinica Universidad de Navarra-Body Adiposity Estimator.

Table 5. Disjoin and join effects of physical activity and the Mediterranean diet in obesity indices according to gender.

\begin{tabular}{|c|c|c|c|}
\hline MALE SUBJECTS & Physical Activity & Mediterranean Diet & $\begin{array}{l}\text { Physical Activity and } \\
\text { Mediterranean Diet }\end{array}$ \\
\hline \multicolumn{4}{|l|}{ Total adiposity } \\
\hline BMI $\left(\mathrm{kg} / \mathrm{m}^{2}\right)$ & 0.003 & 0.774 & 0.509 \\
\hline CUN-BAE (\%) & $<0.001$ & 0.424 & 0.518 \\
\hline Deurenberg (\%) & $<0.001$ & 0.237 & 0.606 \\
\hline \multicolumn{4}{|l|}{ Central adiposity } \\
\hline Waist circumference $(\mathrm{cm})$ & $<0.001$ & 0.697 & 0.538 \\
\hline Conicity index & $<0.001$ & 0.328 & 0.889 \\
\hline Waist to height ratio & $<0.001$ & 0.295 & 0.77 \\
\hline Bonora $\left(\mathrm{cm}^{2}\right)$ & $<0.001$ & 0.721 & 0.584 \\
\hline A body shape index & $<0.001$ & 0.405 & 0.917 \\
\hline Body roundness index & $<0.001$ & 0.287 & 0.783 \\
\hline Neck circumference $(\mathrm{cm})$ & 0.079 & 0.887 & 0.369 \\
\hline \multicolumn{4}{|l|}{ Lean body mass } \\
\hline Hume (kg) & 0.383 & 0.436 & 0.216 \\
\hline \multicolumn{4}{|l|}{ FEMALE SUBJECTS } \\
\hline \multicolumn{4}{|l|}{ Total adiposity } \\
\hline BMI $\left(\mathrm{kg} / \mathrm{m}^{2}\right)$ & 0.061 & 0.435 & 0.808 \\
\hline CUN-BAE (\%) & 0.067 & 0.385 & 0.835 \\
\hline Deurenberg (\%) & 0.045 & 0.304 & 0.837 \\
\hline \multicolumn{4}{|l|}{ Central adiposity } \\
\hline Waist circumference (cm) & 0.01 & 0.161 & 0.335 \\
\hline Conicity index & 0.023 & 0.051 & 0.243 \\
\hline Waist to height ratio & 0.008 & 0.106 & 0.436 \\
\hline Bonora $\left(\mathrm{cm}^{2}\right)$ & 0.007 & 0.044 & 0.442 \\
\hline A body shape index & 0.198 & 0.073 & 0.503 \\
\hline Body roundness index & 0.008 & 0.125 & 0.461 \\
\hline Neck circumference $(\mathrm{cm})$ & 0.192 & 0.508 & 0.612 \\
\hline \multicolumn{4}{|l|}{ Lean body mass } \\
\hline Hume (kg) & 0.39 & 0.295 & 0.511 \\
\hline
\end{tabular}

Two-way ANOVA was used for all $p$-values. BMI: body mass index; CUN-BAE: Clinica Universidad de Navarra-Body Adiposity Estimator. 


\section{Discussion and Conclusions}

The present study compares the impact of physical activity and adherence to MedDiet on different equations used to estimate total body fat and abdominal adiposity in a middle-aged and overweight Caucasian cohort. Our data support the concept that physical activity is related to low adiposity in both genders, showing a positive association in almost all of the assessed indices. However, and contrary to expected, the ability of the MedDiet to modulate the amount of total and central adiposity was small in magnitude. Also, in our population, it is noteworthy that there was no association between combined physical activity and MedDiet adherence and obesity indices.

Our results, together with the continuing worldwide increase in body weight and the favorable impact of regular exercise for reducing the morbidity and mortality associated with cardiovascular disease and diabetes, suggest that the physical activity is a critical behavior among lifestyle strategies, aiming to prevent excess weight and reduce fat mass in overweight subjects [35,36]. In the Look AHEAD trial, among 2240 subjects with type 2 diabetes and obesity, lower physical activity measured with an accelerometry was associated with higher values of BMI [37]. Recently, in a senior Spanish cohort at high cardiovascular disease, greater time spent on moderate to vigorous physical activity and fewer on sedentary behaviors was inversely associated with the prevalence of obesity and some components of the metabolic syndrome [38]. Similarly, in the 2003-2006 National Health and Nutrition Examination Survey, the moderate-to-vigorous physical activity assessed by accelerometers was strongly and negatively associated with BMI and waist circumference, but also with triceps and subscapular skinfolds [39]. However, data about the impact of physical activity on other parameters of total body fat or abdominal adiposity are scarce. Another cross-sectional study of 1009 men aged 71-91 years from primary care centers in the UK showed that each daily additional 30 min of moderate-to-vigorous physical activity was associated with a 0.60 reduction in the fat mass index [40]. More recently, Cameron et al. have demonstrated that moderate-to-vigorous physical activity was negatively associated with percent body fatness and visceral adipose tissue assessed by DXA in 298 overweight adults [41]. In this study, the inverse relationship between physical activity and percentage of body fat was stronger for non-Latinos than for Latinos, a fact that introduces the possibility that differences in diet and eating habits might modulate the impact of physical activity on anthropometric indices [41]. The positive impact of physical activity on weight and abdominal fat reduction also appears without dietary restriction [42,43].

The underlying mechanisms driving the positive impact of physical activity on anthropometric indices are poorly understood, but they might involve the higher sensitivity to lipolytic stimulation and less to insulin suppression of visceral adipose tissue relative to subcutaneous fat $[44,45]$. Also, skeletal muscles secrete interleukin (IL)-6 into the circulatory system during intensive exercise, an interleukin that acts as both a pro-inflammatory cytokine and an anti-inflammatory myokine, which has also been identified as a lipolytic agent [46,47]. Finally, the role of irisin, a novel myokine secreted in response to physical activity, needs to be considered. Irisin exerts its major action by increasing the expression of mitochondrial uncoupling protein 1, which facilitates the conversion of white adipose tissue into beige adipose tissue [48]. Under resting conditions, serum irisin concentrations do not relate to body fat or lean body mass assessed by DXA in healthy humans [49]. However, 8-week endurance-training intervention increased circulating irisin levels in middle-aged adults, and this change occurred concomitantly with reductions in whole-body fat mass and abdominal visceral adipose tissue area [50].

Combined with current evidence, our findings indicate that physical activity needs to be at least moderate in intensity to influence body weight and levels of adiposity. Additionally, preservation of total skeletal muscle mass with a significant reduction in total and abdominal adipose tissue measured by magnetic resonance imaging was detected independent of exercise amount or intensity in 103 adults with abdominal obesity [51]. Similarly, our data also failed to detect differences in lean body mass across physical activity categories. 
MedDiet is a dietary pattern that has been praised for its success in promoting weight loss and reducing long-term weight gain if energy restriction exists [52]. However, contradictory findings have been reported between MedDiet adherence and changes in weight or overweight/obesity risk [53,54]. The number of calories of MedDiet components, such as olive oil, whole cereals, and nuts, might explain why we failed to detect differences in obesity indices across MedDiet categories, suggesting that participants in the ILERVAS trial with higher adherence to MedDiet failed to achieve a negative energy balance. In fact, the traditional nutritional pattern that characterizes MedDiet can exert its effect through dissimilar pathophysiological mechanisms not related with weight loss, such as improving the lipid profile, modulating inflammation, enhancing its anti-oxidant properties, and reducing blood pressure and insulin resistance, among others [55,56].

Randomized clinical trials and meta-analyses support that physical activity added to dietary intervention further enhances the amount of weight loss achieved [57]. Contrary to expected, our findings in a large overweight population with moderate cardiovascular risk failed to demonstrate a positive interaction between physical activity practice and MedDiet adherence and adiposity indices. It is well known that lifestyle interventions on diet and exercise promotion are especially effective at reducing weight in those individuals with obesity, whereas participants in the ILERVAS project were especially overweight.

Potential limitations must be highlighted in our study. First, we lack the true percentage and distribution of body fat determined with a gold standard tool. Also, we have not evaluated other obesity indices that include the hip circumference, such as abdominal volume index, body adiposity index, and waist to hip ratio. Therefore, we are not sure whether the inclusion of hip circumference would modify our results. However, the close relationship between obesity indices and adiposity has been well established, and remarkable differences between these were not detected in our large population. Second, as an observational and cross-sectional study, a causal relationship between physical activity practice and adherence to the Mediterranean dietary pattern and excess body adiposity cannot be established. Third, the time of physical activity and food consumption was self-reported by participants. Since the study did not use the triaxial accelerometer or doubly labeled water to accurately determine physical activity level or energy expenditure, neither a weighing dietary record to obtain a detailed dietary pattern, this is an important limitation of the study. Fourth, information about socio-demographic and lifestyle characteristics, which have been related to body composition, was not available from the participants included in the study, as well as information about whether participants performed hypocaloric diets or had taken protein supplements in previous months [58,59]. Finally, results from this study cannot be extrapolated to other populations, since our population comprised of Spanish middle-age individuals that were overweight and at low-to-moderate cardiovascular risk.

In conclusion, our records demonstrate that moderate to vigorous physical activity practice is related to lower obesity indices in a large sample of middle-aged subjects. However, MedDiet showed a slight impact on anthropometric indices. There are also no joint associations between physical activity and MedDiet with lower adiposity measurements. In a world, where most adults are performing less than the recommended intensities of physical activity, our results encourage the inclusion of physical activity as an important lifestyle behavior for regulating body composition and minimizing the comorbidities associated with excess body fat [60].

Supplementary Materials: The following are available online at http://www.mdpi.com/2072-6643/11/6/1359/s1, Figure S1: Flow chart of the study population.

Author Contributions: Conceptualization, A.L., M.S., E.S.; Data curation, M.S., E.S., M.M.-A., E.M., M.P.-O., and M.J.; Formal analysis M.S., E.S., A.L., and G.T.; Funding acquisition, E.F.; Investigation, M.S., E.S., and A.L.; Methodology, M.H., J.G., F.P., F.R., R.P., C.F.-S., F.B., G.A., A.V., S.B., M.O.-B, and L.G.-C.; Project administration, A.L.; Resources, E.F.; Supervision, M.B.-L., J.S., J.M.V., P.G., M.J., M.M.-A., E.M., E.C., and J.S.-S.; Visualization, M.S., E.S., S.C.; Writing-original draft, M.S., E.S.; Writing—review \& editing, A.L. All authors approved the final version of the manuscript to be published. 
Funding: This study was partially supported by grants from the Diputació de Lleida, Generalitat de Catalunya (2017SGR696 and SLT0021600250). CIBER de Diabetes y Enfermedades Metabólicas Asociadas, CIBER de Nutrición y Obesidad, and CIBER de Enfermedades Respiratorias are initiatives of the Instituto de Salud Carlos III.

Acknowledgments: The authors would also like to thank Fundació Renal Jaume Arnó, the nurses' staff, and the Primary Care members for recruiting subjects and their work in the accurate progress of the ILERVAS project (see Appendix A).

Conflicts of Interest: The authors declare no conflicts of interest.

\section{Appendix A}

Author List and Affiliations of ILERVAS Project collaborators:

Ferran Barbé 1,2, José Manuel Valdivielso ${ }^{3}$, Gloria Arqué 4, Silvia Barril 1,2, Ana Vena 4, Pere Godoy ${ }^{5,6}$, Mariona Jové ${ }^{7}$, Montserrat Martínez-Alonso ${ }^{8}$, Eva Miquel ${ }^{9,10}$, Marta Ortega-Bravo ${ }^{5,9}$, Manel Portero-Otin ${ }^{7}$, Serafín Cambray ${ }^{3}$, Gerard Torres ${ }^{1,2}$ and Eva Castro ${ }^{3}$

1 Respiratory Department, Arnau de Vilanova-Santa María University Hospital, Translational Research in Respiratory Medicine, IRBLleida, University of Lleida, Lleida, Spain.

2 Centro de Investigación Biomédica en Red de Enfermedades Respiratorias (CIBERES), Instituto de Salud Carlos III (ISCIII), Madrid, Spain.

3 Vascular and Renal Translational Research Group, IRBLleida, RedinRen-ISCIII, Lleida, Spain.

4 Stroke Unit. University Hospital Arnau de Vilanova, Clinical Neurosciences Group, IRBLleida, University of Lleida, Lleida, Spain.

5 Applied Epidemiology Research Group, IRBLleida, Agència de Salut Pública de Catalunya, Lleida, Spain.

6 Centro de Investigación Biomédica en Red de Epidemiología y Salud Pública (CIBERESP), Instituto de Salud Carlos III (ISCIII).

7 Department of Experimental Medicina, IRBLleida, University of Lleida, Lleida, Spain.

8 Systems Biology and Statistical Methods for Biomedical Research Group, Biostatistics Unit, IRBLleida, Universitat de Lleida, Lleida, Spain.

9 Unitat de Suport a la Recerca Lleida, Fundació Institut Universitari per a la recerca a l'Atenció Primària de Salut Jordi Gol i Gurina (IDIAPJGol), Barcelona, Spain.

\section{References}

1. Inoue, Y.; Qin, B.; Poti, J.; Sokol, R.; Gordon-Larsen, P. Epidemiology of obesity in adults: Latest trends. Curr. Obes. Rep. 2018, 7, 276-288. [CrossRef] [PubMed]

2. Pearson, N.; Biddle, S.J. Sedentary behavior and dietary intake in children, adolescents, and adults. A systematic review. Am. J. Prev. Med. 2011, 41, 178-188. [CrossRef]

3. World Health Organization (WHO). Global Health Observatory (GHO) Data. Obesity. Available online: https://www.who.int/features/factfiles/obesity/en/ (accessed on 15 October 2017).

4. Upadhyay, J.; Farr, O.; Perakakis, N.; Ghaly, W.; Mantzoros, C. Obesity as a disease. Med. Clin. N. Am. 2018, 102, 13-33. [CrossRef] [PubMed]

5. Mohammadi, H.R.; Khoshnam, M.S.; Khoshnam, E. Effects of different modes of exercise training on body composition and risk factors for cardiovascular disease in middle-aged men. Int. J. Prev. Med. 2018, 9, 9. [PubMed]

6. Drenowatz, C.; Shook, R.P.; Hand, G.A.; Hébert, J.R.; Blair, S.N. The independent association between diet quality and body composition. Sci. Rep. 2014, 4, 4928. [CrossRef] [PubMed]

7. Castro-Quezada, I.; Román-Viñas, B.; Serra-Majem, L. The mediterranean diet and nutritional adequacy: A review. Nutrients 2014, 6, 231-248. [CrossRef] [PubMed]

8. Davis, C.; Bryan, J.; Hodgson, J.; Murphy, K. Definition of the Mediterranean Diet: A Literature Review. Nutrients 2015, 7, 9139-9153. [CrossRef]

9. Serra-Majem, L.; Roman, B.; Estruch, R. Scientific evidence of interventions using the Mediterranean diet: A systematic review. Nutr. Rev. 2006, 64, S27-S47. [CrossRef]

10. Godos, J.; Zappalà, G.; Bernardini, S.; Giambini, I.; Bes-Rastrollo, M.; Martinez-Gonzalez, M. Adherence to the Mediterranean diet is inversely associated with metabolic syndrome occurrence: A meta-analysis of observational studies. Int. J. Food Sci. Nutr. 2017, 68, 138-148. [CrossRef] 
11. Valls-Pedret, C.; Sala-Vila, A.; Serra-Mir, M.; Corella, D.; De la Torre, R.; Martínez-González, M.Á. Mediterranean diet and age-related cognitive decline: A randomized clinical trial. JAMA Intern. Med. 2015, 175, 1094-1103. [CrossRef]

12. Estruch, R.; Ros, E.; Salas-Salvadó, J.; Covas, M.I.; Corella, D.; Arós, F.; Gómez-Gracia, E.; Ruiz-Gutiérrez, V.; Fiol, M.; Lapetra, J.; et al. Primary prevention of cardiovascular disease with a Mediterranean diet supplemented with extra-virgin olive oil or nuts. N. Engl. J. Med. 2018, 378, 1279-1290. [CrossRef] [PubMed]

13. Itsiopoulos, C.; Kucianski, T.; Mayr, H.L.; van Gaal, W.J.; Martinez-Gonzalez, M.A.; Vally, H.; Kingsley, M.; Kouris-Blazos, A.; Radcliffe, J.; Segal, L.; et al. The AUStralian MEDiterranean diet heart trial (AUSMED heart trial): A randomized clinical trial in secondary prevention of coronary heart disease in a multi-ethnic Australian population: Study protocol. Am. Heart J. 2018, 203, 4-11. [CrossRef] [PubMed]

14. Boghossian, N.S.; Yeung, E.H.; Mumford, S.L.; Zhang, C.; Gaskins, A.J.; Wactawski-Wende, J.; Schisterman, E.F.; BioCycle Study Group. Adherence to the Mediterranean diet and body fat distribution in reproductive aged women. Eur. J. Clin. Nutr. 2013, 67, 289-294. [CrossRef] [PubMed]

15. Bertoli, S.; Leone, A.; Vignati, L.; Bedogni, G.; Martínez-González, M.Á.; Bes-Rastrollo, M.; Spadafranca, A.; Vanzulli, A.; Battezzati, A. Adherence to the Mediterranean diet is inversely associated with visceral abdominal tissue in Caucasian subjects. Clin. Nutr. 2015, 34, 1266-1272. [CrossRef] [PubMed]

16. Barrea, L.; Muscogiuri, G.; Macchia, P.E.; Di Somma, C.; Falco, A.; Savanelli, M.C.; Colao, A.; Savastano, S. Mediterranean Diet and Phase Angle in a Sample of Adult Population: Results of a Pilot Study. Nutrients 2017, 9, 151. [CrossRef] [PubMed]

17. Blundell, J.E.; Dulloo, A.G.; Salvador, J.; Frühbeck, G.; EASO SAB Working Group on BMI. Beyond BMI-Phenotyping the obesities. Obes. Facts 2014, 7, 322-328. [CrossRef]

18. Gómez-Ambrosi, J.; Silva, C.; Galofré, J.C.; Escalada, J.; Santos, S.; Millán, D.; Vila, N.; Ibañez, P.; Gil, M.J.; Valentí, V.; et al. Body mass index classification misses subjects with increased cardiometabolic risk factors related to elevated adiposity. Int. J. Obes. 2012, 36, 286-294. [CrossRef]

19. Kelly, T.L.; Wilson, K.E.; Heymsfield, S.B. Dual energy X-Ray absorptiometry body composition reference values from NHANES. PLoS ONE 2009, 4, e7038. [CrossRef]

20. Gómez-Ambrosi, J.; Silva, C.; Catalán, V.; Rodríguez, A.; Galofré, J.C.; Escalada, J.; Valentí, V.; Rotellar, F.; Romero, S.; Ramírez, B.; et al. Clinical usefulness of a new equation for estimating body fat. Diabetes Care 2012, 35, 383-388. [CrossRef]

21. Deurenberg, P.; Weststrate, J.A.; Seidell, J.C. Body mass index as a measure of body fatness: Age- and sex-specific prediction formulas. Br. J. Nutr. 1991, 65, 105-114. [CrossRef]

22. Valdez, R. A simple model-based index of abdominal adiposity. J. Clin. Epidemiol. 1991, 44, $955-956$. [CrossRef]

23. Ashwell, M.; Gunn, P.; Gibson, S. Waist-to-height ratio is a better screening tool than waist circumference and BMI for adult cardiometabolic risk factors: Systematic review and meta-analysis. Obes. Rev. 2012, 13, 275-286. [CrossRef] [PubMed]

24. Bonora, E.; Micciolo, R.; Ghiatas, A.A.; Lancaster, J.L.; Alyassin, A.; Muggeo, M.; DeFronzo, R.A. Is it possible to derive a reliable estimate of human visceral and subcutaneous abdominal adipose tissue from simple anthropometric measurements? Metabolism 1995, 44, 1617-1625. [CrossRef]

25. Krakauer, N.Y.; Krakauer, J.C. A new body shape index predicts mortality hazard independently of body mass index. PLoS ONE 2012, 7, e39504. [CrossRef] [PubMed]

26. Thomas, D.M.; Bredlau, C.; Bosy-Westphal, A.; Mueller, M.; Shen, W.; Gallagher, D.; Maeda, Y.; McDougall, A.; Peterson, C.M.; Ravussin, E.; et al. Relationships between body roundness with body fat and visceral adipose tissue emerging from a new geometrical model. Obesity 2013, 21, 2264-2271. [CrossRef] [PubMed]

27. Després, J.P.; Couillard, C.; Gagnon, J.; Bergeron, J.; Leon, A.S.; Rao, D.C.; Skinner, J.S.; Wilmore, J.H.; Bouchard, C. Race, visceral adipose tissue, plasma lipids, and lipoprotein lipase activity in men and women: The Health, Risk Factors, Exercise Training, and Genetics (HERITAGE) family study. Arterioscler. Thromb. Vasc. Biol. 2000, 20, 1932-1938. [CrossRef] [PubMed]

28. Betriu, À.; Farràs, C.; Abajo, M.; Martinez-Alonso, M.; Arroyo, D.; Barbé, F.; Buti, M.; Lecube, A.; Portero, M.; Purroy, F.; et al. Randomised intervention study to assess the prevalence of subclinical vascular disease and hidden kidney disease and its impact on morbidity and mortality: The ILERVAS project. Nefrologia 2016, 36, 389-396. [CrossRef] [PubMed] 
29. Schröder, H.; Fitó, M.; Estruch, R.; Martínez-González, M.A.; Corella, D.; Salas-Salvadó, J.; Lamuela-Raventós, R.; Ros, E.; Salaverría, I.; Fiol, M.; et al. A short screener is valid for assessing mediterranean diet adherence among older Spanish men and women. J. Nutr. 2011, 14, 1140-1145. [CrossRef]

30. Craig, C.L.; Marshall, A.L.; Sjöström, M.; Bauman, A.E.; Booth, M.L.; Ainsworth, B.E.; Pratt, M.; Ekelund, U.; Yngve, A.; Sallis, J.F.; et al. International physical activity questionnaire: 12-country reliability and validity. Med. Sci. Sports Exerc. 2003, 35, 1381-1395. [CrossRef]

31. World Health Organization. Obesity: Preventing and Managing the Global Epidemic; Report of a WHO Consultation; WHO Technical Report Series; World Health Organization: Geneva, Switzerland, 2000; pp. 1-252.

32. Ma, W.Y.; Yang, C.Y.; Shih, S.R.; Hsieh, H.J.; Hung, C.S.; Chiu, F.C.; Lin, M.S.; Liu, P.H.; Hua, C.H.; Hsein, Y.C.; et al. Measurement of Waist Circumference: Midabdominal or iliac crest? Diabetes Care 2013, 36, 1660-1666. [CrossRef]

33. Ben-Noun, L.L.; Laor, A. Relationship between changes in neck circumference and cardiovascular risk factors. Exp. Clin. Cardiol. 2006, 11, 14-20. [PubMed]

34. Hume, R. Prediction of lean body mass from height and weight. J. Clin. Pathol. 1966, 19, 389-391. [CrossRef] [PubMed]

35. Nystoriak, M.A.; Bhatnagar, A. Cardiovascular effects and benefits of exercise. Front. Cardiovasc. Med. 2018, 5, 135. [CrossRef] [PubMed]

36. Villareal, D.; Aguirre, L.; Gurney, B.; Waters, D.L.; Sinacore, D.R.; Colombo, E.; Armamento-Villareal, R.; Qualls, C. Aerobic or resistance exercise, or both, in dieting obese older adults. N. Engl. J. Med. 2017, 376, 1943-1955. [CrossRef] [PubMed]

37. Jakicic, J.M.; Gregg, E.; Knowler, W.; Kelley, D.E.; Lang, W.; Miller, G.D.; Pi-Sunyer, F.X.; Regensteiner, J.G.; Rejeski, W.J.; Ridisl, P.; et al. Activity patterns of obese adults with type 2 diabetes in the Look AHEAD study. Med. Sci. Sports Exerc. 2010, 42, 1995-2005. [CrossRef]

38. Rosique-Esteban, N.; Díaz-López, A.; Martínez-González, M.A.; Corella, D.; Goday, A.; Martínez, J.A.; Romaguera, D.; Vioque, J.; Arós, F.; Garcia-Rios, A.; et al. Leisure-time physical activity, sedentary behaviors, sleep, and cardiometabolic risk factors at baseline in the PREDIMED-PLUS intervention trial: A cross-sectional analysis. PLoS ONE 2017, 12, e0172253. [CrossRef]

39. Wolff-Hughes, D.L.; Fitzhugh, E.C.; Bassett, D.R.; Churilla, J.R. Total activity counts and bouted minutes of moderate-to-vigorous physical activity: Relationships with cardiometabolic biomarkers using 2003-2006 NHANES. J. Phys. Act Health 2015, 12, 694-700. [CrossRef]

40. Jefferis, B.J.; Parsons, T.J.; Sartini, C.; Ash, S.; Lennon, L.T.; Wannamethee, S.G.; Lee, I.M.; Whincup, P.H. Does duration of physical activity bouts matter for adiposity and metabolic syndrome? A cross-sectional study of older British men. Int. J. Behav. Nutr. Phys. Act. 2016, 13, 36. [CrossRef]

41. Cameron, N.; Godino, J.; Nichols, J.F.; Wing, D.; Hill, L.; Patrick, K. Associations between physical activity and BMI, body fatness, and visceral adiposity in overweight or obese Latino and non-Latino adults. Int. J. Obes. 2017, 41, 873-877. [CrossRef]

42. Ross, R.; Dagnone, D.; Jones, P.J.; Smith, H.; Paddags, A.; Hudson, R.; Janssen, I. Reduction in obesity and related comorbid conditions after diet-induced weight loss or exercise-induced weight loss in men. Ann. Intern. Med. 2000, 133, 92-103. [CrossRef]

43. Mourier, A.; Gautier, J.F.; De Kerviler, E.; Bigard, A.X.; Villette, J.M.; Garnier, J.P.; Duvallet, A.; Guezennec, C.Y.; Cathelineau, G. Mobilization of visceral adipose tissue related to the improvement in insulin sensitivity in response to physical training in NIDDM. Effects of branched-chain amino acid supplements. Diabetes Care 1997, 20, 385-391. [CrossRef] [PubMed]

44. Fried, S.K.; Leibel, R.L.; Edens, N.K.; Kral, J.G. Lipolysis in intraabdominal adipose tissue of obese women and men. Obes. Res. 1993, 1, 443-448. [CrossRef] [PubMed]

45. Meek, S.E.; Nair, K.S.; Jensen, M.D. Insulin regulation of regional free fatty acid metabolism. Diabetes 1999, 48, 10-14. [CrossRef] [PubMed]

46. Petersen, A.M.W.; Pedersen, B.K. The role of IL-6 in mediating the anti-inflammatory effects of exercise. J. Physiol. Pharmacol. 2006, 57 (Suppl. 10), 43-51. [PubMed]

47. Petersen, E.W.; Carey, A.L.; Sacchetti, M.; Steinberg, G.R.; Macaulay, S.L.; Febbraio, M.A.; Pedersen, B.K. Acute IL-6 treatment increases fatty acid turnover in elderly humans in vivo and in tissue culture in vitro. Am. J. Physiol. Endocrinol. Metab. 2005, 288, 155-162. [CrossRef] 
48. Buscemi, S.; Corleo, D.; Vasto, S.; Buscemi, C.; Massenti, M.F.; Nuzzo, D.; Lucisano, G.; Barile, A.M.; Rosafio, G.; Maniaci, V.; et al. Factors associated with circulating concentrations of irisin in the general population cohort of the ABCD study. Int. J. Obes. 2018, 42, 398-404. [CrossRef] [PubMed]

49. Biniaminov, N.; Bandt, S.; Roth, A.; Haertel, S.; Neumann, R.; Bub, A. Irisin, physical activity and fitness status in healthy humans: No association under resting conditions in a cross-sectional study. PLoS ONE 2018, 13, e0189254. [CrossRef]

50. Miyamoto-Mikami, E.; Sato, K.; Kurihara, T.; Hasegawa, N.; Fujie, S.; Fujita, S.; Sanada, K.; Hamaoka, T.; Tabata, I.; Iemitsu, M. Endurance training-induced increase in circulating irisin levels is associated with reduction of abdominal visceral fat in middle-aged and older adults. PLoS ONE 2015, 10, e0120354. [CrossRef]

51. Cowan, T.E.; Brennan, A.M.; Stotz, P.J.; Clarke, J.; Lamarche, B.; Ross, R. Separate Effects of Exercise Amount and Intensity on Adipose Tissue and Skeletal Muscle Mass in Adults with Abdominal Obesity. Obesity 2018, 26, 1696-1703. [CrossRef]

52. Ulian, M.D.; Aburad, L.; da Silva Oliveira, M.S.; Poppe, A.C.M.; Sabatini, F.; Perez, I.; Gualano, B.; Benatti, F.B.; Pinto, A.J.; Roble, O.J.; et al. Effects of health at every size ${ }^{\circledR}$ interventions on health-related outcomes of people with overweight and obesity: A systematic review. Obes. Rev. 2018, 19, 1659-1666. [CrossRef]

53. Anton, S.D.; Hida, A.; Heekin, K.; Sowalsky, K.; Karabetian, C.; Mutchie, H.; Leeuwenburgh, C.; Manini, T.M.; Barnett, T.E. Effects of Popular Diets without Specific Calorie Targets on Weight Loss Outcomes: Systematic Review of Findings from Clinical Trials. Nutrients 2017, 9, 822. [CrossRef] [PubMed]

54. Mancini, J.G.; Filion, K.B.; Atallah, R.; Eisenberg, M.J. Systematic Review of the Mediterranean Diet for Long-Term Weight Loss. Am. J. Med. 2016, 129, 407-415. [CrossRef] [PubMed]

55. Casas, R.; Sacanella, E.; Estruch, R. The immune protective effect of the Mediterranean diet against chronic low-grade inflammatory diseases. Endocr. Metab. Immune Disord. Drug Targets 2014, 14, 245-254. [CrossRef] [PubMed]

56. Salas-Salvadó, J.; Becerra-Tomás, N.; García-Gavilán, J.F.; Bulló, M.; Barrubés, L. Mediterranean diet and cardiovascular disease prevention: What do we know? Prog. Cardiovasc. Dis. 2018, 61, 62-67. [CrossRef] [PubMed]

57. Goodpaster, B.H.; DeLany, J.P.; Otto, A.D.; Kuller, L.; Vockley, J.; South-Paul, J.E.; Thomas, S.B.; Brown, J.; McTigue, K.; Hames, K.C.; et al. Effects of diet and physical activity interventions on weight loss and cardiometabolic risk factors in severely obese adults: A randomized trial. JAMA 2010, 304, 1795-1802. [CrossRef] [PubMed]

58. Nang, E.E.; van Dam, R.M.; Tan, C.S.; Mueller-Riemenschneider, F.; Lim, Y.T.; Ong, K.Z.; Ee, S.; Lee, J.; Tai, E.S. Association of television viewing time with body composition and calcified subclinical atherosclerosis in Singapore Chinese. PLOS ONE 2015, 10, e0132161. [CrossRef]

59. López-Sobaler, A.M.; Rodríguez-Rodríguez, E.; Aranceta-Bartrina, J.; Gil, Á.; González-Gross, M.; Serra-Majem, L.; Varela-Moreiras, G.; Ortega, R.M. General and abdominal obesity is related to physical activity, smoking and sleeping behaviours and mediated by the educational level: Findings from the ANIBES Study in Spain. PLoS ONE 2016, 11, e0169027. [CrossRef]

60. Ozemek, C.; Lavie, C.J.; Rognmo, Ø. Global physical activity levels-Need for intervention. Prog. Cardiovasc. Dis. 2019, 62, 102-107. [CrossRef]

(C) 2019 by the authors. Licensee MDPI, Basel, Switzerland. This article is an open access article distributed under the terms and conditions of the Creative Commons Attribution (CC BY) license (http://creativecommons.org/licenses/by/4.0/). 